\title{
NMR solution structure of a parallel LNA quadruplex
}

\author{
Antonio Randazzo, Veronica Esposito, Oliver Ohlenschläger ${ }^{1}$, \\ Ramadurai Ramachandran ${ }^{1}$ and Luciano Mayol*
}

Dipartimento di Chimica delle Sostanze Naturali, Università degli Studi di Napoli 'Federico II', via D. Montesano 49, I-80131 Napoli, Italy and ${ }^{1}$ Institut für Molekulare Biotechnologie, Abt. Molekulare Biophysik/NMR-Spektroskopie, Beutenbergstr. 11, D-07745 Jena, Germany

Received January 28, 2004; Revised and Accepted May 11, 2004

\begin{abstract}
The solution structure of a locked nucleic acid (LNA) quadruplex, formed by the oligomer d(TGGGT), containing only conformationally restricted LNA residues is reported. NMR and CD spectroscopy, as well as molecular dynamics and mechanic calculations, has been used to characterize the complex. The molecule adopts a parallel stranded conformation with a 4-fold rotational symmetry, showing a right-handed helicity and the guanine residues in an almost planar conformation with three well-defined G-tetrads. The thermal stability of Q-LNA has been found to be comparable with that of $[\mathrm{r}(\mathrm{UGGGU})]_{4}$, while a $T_{\mathrm{m}}$ increment of $20^{\circ} \mathrm{C}$ with respect to the corresponding DNA quadruplex structure $[\mathrm{d}(\mathrm{TGGGT})]_{4}$ has been observed. The structural features of the LNA quadruplex reported here may open new perspectives for the biological application of LNAs as novel versatile tools to design aptamer or catalyst oligonucleotides.
\end{abstract}

\section{INTRODUCTION}

In recent years, considerable efforts have been dedicated to the synthesis and investigation of new DNA analogs with improved binding properties towards nucleic acids than the canonical counterparts. In 1988 the first oligonucleotides containing one or more $2^{\prime}-\mathrm{O}, 4^{\prime}-\mathrm{C}$ methylene-linked bicyclic ribonucleosides (LNAs, locked nucleic acids) (Fig. 1) were prepared by the groups of Wengel (1) and Imanishi (2). They also described the unprecedented hybridization affinity of LNAs towards complementary nucleic acids. In LNA residues the distinctive conformational restriction arising from the $2^{\prime}-\mathrm{O}, 4^{\prime}-\mathrm{C}$ methylene bridge is mainly responsible for their enhanced hybridization performance (3) and for their exceptional biostability (4). As a matter of fact, the methylene bridge confers an RNA-like C3'-endo (N-type) conformation to the sugar moiety of the modified nucleotide and reduces its conformational flexibility, thus expanding the degree of local organization of the phosphate backbone $(5,6)$. This entropic constraint leads to an enhanced affinity towards complementary RNA and DNA targets, with an increase from 4.0 to $9.3^{\circ} \mathrm{C}$ in thermal stability of the resulting heteroduplexes per introduced LNA monomer (7).

The unprecedented hybridization properties suggest that LNAs could be powerful agents for fine tuning drugs with a very specific target potential, thus providing a new class of therapeutics (8-10). Actually, LNAs represent very versatile tools for the control of gene expression, the treatment of various human diseases and diagnostic assays. In fact, chimeric $2^{\prime}$ - $O$-methyl/LNA oligoribonucleotides were found to inhibit RNA-protein interactions important for HIV replication by sterically blocking the trans-activation responsive region TAR (11). Furthermore, incorporation of LNA monomers into the binding arms of the '10-23' DNAzyme, yielding an LNAzyme, markedly increases cleavage properties towards the target RNA (12). For antisense applications of LNAs, it is important to note that the introduction of just two or three LNA monomers in a DNA strand, or the use of LNA/ DNA mixmers also elicited RNase $\mathrm{H}$ activity (13): the LNA bases confer enhanced binding to mRNA target, while DNA bases create a suitable RNA-DNA hybrid to act as a substrate for RNase H. Furthermore, homopyrimidine LNAs can also bind to duplex DNA by triple helix formation $(14,15)$, even at neutral $\mathrm{pH}$. Central incorporation of one LNA monomer in TFOs (triplex forming oligonucleotides) significantly increased the melting temperatures of the resulting triplexes by $4-5^{\circ} \mathrm{C}(15)$, constituting a possible avenue for gene regulation.

In view of the ability of short LNAs to stably bind complementary sequences, it is possible to use them for competitive blocking of the RNA template component of human telomerase (16). This RNA domain contains an $11 \mathrm{mer}$ sequence that binds telomeric DNA and guides the addition of telomeric repeats, constituting an attractive target for oligonucleotide-induced inhibition. Elayadi et al. (16) have targeted the telomerase template with LNA oligonucleotides and found them to act as potent and selective inhibitors. Another interesting application of LNAs is represented by strategies targeting transcription-activating proteins. Double stranded DNAs including the consensus binding sequence of a specific transcription factor can act as decoy, inactivating this target protein and, hence, blocking the transcription of the

*To whom correspondence should be addressed. Tel: +39 081 678508; Fax: +39 081 678552; Email: mayoll@unina.it 


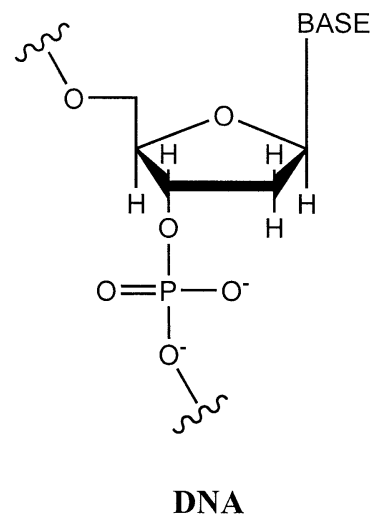

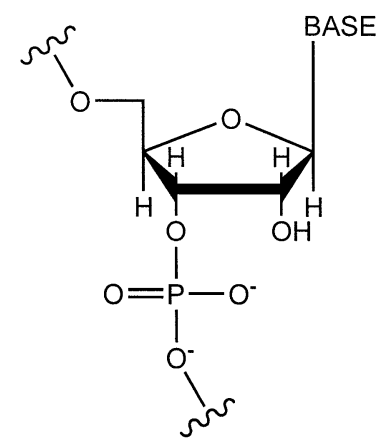

RNA

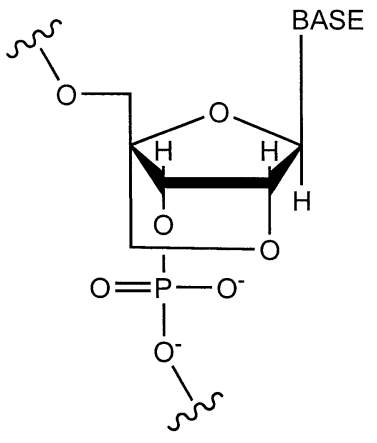

LNA

Figure 1. Chemical structure of DNA, RNA and LNA.

corresponding gene (17). Incorporation of one or two terminal LNA monomers outside the transcription factor $\kappa \mathrm{B}(\mathrm{NF}-\mathrm{\kappa B})$ sequence markedly increased the stability towards nuclease digestion without interfering with $\mathrm{\kappa B}$ interaction (18). This clearly indicates that LNAs can operate as aptamers and that it could be possible, at least in principle, to design NF- $\kappa B$ LNA decoys provided with excellent nuclease stability and still capable to be efficiently recognized by the protein.

On the contrary, very little is known about the capability of LNAs to form quadruplex structures (19). DNA and RNA quadruplex structures contain stacks of cyclic hydrogenbonded arrays of four co-planar guanine bases (G-quartets). Particularly, G-rich sequences can fold in a number of different ways, as far as strand stoichiometry and orientation are concerned. Strand stoichiometry variation allows Gquadruplexes to be formed by association of one, via intramolecular folding (20-22), two, by dimerization of a folded-back hairpin $(23,24)$, or four separate strands $(25-27)$. These structures have recently received considerable attention as G-rich sequences are found in a number of biologically important DNA regions, including telomeres $(28,29)$, the immunoglobuline switch region (30), the c-myc promoter (31) and in other hot spots for genetic recombination (32). Moreover, some examples exist where aptameric nucleic acids possess quadruplex structures (33). The biological relevance of quadruplexes has already led to the use of nucleic acid analogs such as peptidic nucleic acids (PNAs) in order to improve their physical and biological properties. Very recently, several studies about the formation of PNA/DNA hybrid quadruplexes confirmed the ability of PNA to mimic DNA even in more complex multi-stranded structures $(34,35)$. However, the poor water solubility of PNAs limits their potential applications.

In light of the above and considering the advantageous characteristics of LNAs, such as high water solubility, easy handling and synthetic accessibility through the conventional phosphoramidite chemistry, we have undertaken a study concerning the capability of these nucleic acid analogs to form quadruplex structures.

In this paper we report the structural characterization, based on NMR and CD spectroscopy, associated with molecular mechanics and dynamics calculations, of a parallel stranded quadruplex formed by the sequence 5'-(TGGGT)-3' ('Q-LNA' in short), containing all LNA monomers.

\section{MATERIALS AND METHODS}

\section{LNA and DNA oligonucleotides}

The pentanucleotide $5^{\prime}$-(TGGGT)-3' (54 O.D.), containing all LNA residues, was purchased from Exiqon AS (Vedbaek, Denmark) and successively desalted by Sep-Pak cartridges (C18).

The pentanucleotide $5^{\prime}$-r(UGGGU)-3' (26 O.D.), was purchased from Primm (Milano, Italy).

The regular DNA oligonucleotide d(TGGGT) was synthesized on a Millipore Cyclon Plus DNA synthesizer, using solid phase $\beta$-cyanoethyl phosphoramidite chemistry at $15 \mu \mathrm{mol}$ scale. The oligomer was detached from the support and deprotected by treatment with conc. aqueous ammonia at $55^{\circ} \mathrm{C}$ for $12 \mathrm{~h}$. The combined filtrates and washings were concentrated under reduced pressure, redissolved in $\mathrm{H}_{2} \mathrm{O}$ and analysed and purified by HPLC on a Nucleogel SAX column (1000-8/46, Macherey-Nagel, Düren, Germany); using buffer A: $20 \mathrm{mM} \mathrm{KH}_{2} \mathrm{PO}_{4}$ aq. solution, $\mathrm{pH} 7.0$, containing $20 \%(\mathrm{v} / \mathrm{v})$ $\mathrm{CH}_{3} \mathrm{CN}$; buffer B: $1 \mathrm{M} \mathrm{KCl}, 20 \mathrm{mM} \mathrm{KH_{2 }} \mathrm{PO}_{4}$ aq. solution, $\mathrm{pH} 7.0$, containing $20 \%$ (v/v) $\mathrm{CH}_{3} \mathrm{CN}$; a linear gradient from 0 to $100 \% \mathrm{~B}$ in $30 \mathrm{~min}$ and flow rate $1 \mathrm{ml} / \mathrm{min}$ were used.

All oligomers were $>98 \%$ pure [nuclear magnetic resonance (NMR)].

\section{Nuclear magnetic resonance}

The NMR sample had a concentration of $\sim 1.80 \mathrm{mM}$, in $0.6 \mathrm{ml}$

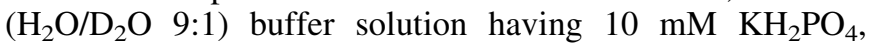
$70 \mathrm{mM} \mathrm{KCl}, 0.2 \mathrm{mM}$ EDTA, pH 7.0. For $\mathrm{D}_{2} \mathrm{O}$ experiments, the $\mathrm{H}_{2} \mathrm{O}$ was replaced by drying down the sample, lyophilization and redissolution in $\mathrm{D}_{2} \mathrm{O}$. NMR spectra were recorded with Bruker AMX 500, Varian Unity INOVA 600 and $750 \mathrm{MHz}$ spectrometers at $27^{\circ} \mathrm{C} .{ }^{1} \mathrm{H}$ chemical shifts were referenced relative to external sodium 2,2-dimethyl-2-silapentane-5sulfonate, whereas ${ }^{31} \mathrm{P}$ chemical shifts were referenced to external phosphoric acid $\left(\mathrm{H}_{3} \mathrm{PO}_{4} 85 \% \mathrm{v} / \mathrm{v}\right)$. 1D proton spectra of samples in $\mathrm{H}_{2} \mathrm{O}$ were recorded using the watergate sequence (36). A proton-detected ${ }^{1} \mathrm{H}^{3}{ }^{31} \mathrm{P}$ heteronuclear COSY was recorded in $\mathrm{D}_{2} \mathrm{O}$ in the hypercomplex mode with 
$2048 t_{2}$ points and $96 t_{1}$ increments, and a spectral width of $500 \mathrm{~Hz}$ along the ${ }^{31} \mathrm{P}$ dimension. Phase sensitive NOESY spectra (37) were recorded with mixing times of 100 and $180 \mathrm{~ms}\left(\mathrm{~T}=27^{\circ} \mathrm{C}\right)$. The watergate (36) technique was also used for acquiring NOESY spectra in $\mathrm{H}_{2} \mathrm{O}$. TOCSY spectra (38) with mixing times of $120 \mathrm{~ms}$ were recorded with $\mathrm{D}_{2} \mathrm{O}$ solution. NOESY and TOCSY were recorded using the TPPI (39) procedure for quadrature detection. In all 2D experiments the time domain data consisted of 2048 complex points in $t_{2}$ and 400-512 FIDs in $t_{1}$ dimension. The relaxation delay was kept at $1.2 \mathrm{~s}$ for all experiments. The NMR data were processed on a SGI Octane workstation using FELIX 98 software (Accelrys, San Diego, CA).

\section{Structure calculations}

The structure calculations were performed with the program CYANA (40) starting from 200 random conformations. Upper limit distance constraints for both exchangeable and nonexchangeable hydrogens were classified according to the intensity of the cross peaks in the NOESY spectra (with $180 \mathrm{~ms}$ mixing time) with the CALIBA tool of the program CYANA (40). Pseudo-atoms were introduced where needed. 544 upper distance restraints were calculated (136 per strand) and reduced to 292 (73 per strand) after removal of irrelevant restraints. Hydrogen bond constraints (16 upper and 16 lower limit constraints/G-tetrad) were incorporated with upper and lower distance limits of 2.0 and $1.7 \AA$ for the hydrogenacceptor distance and 3.0 and $2.7 \AA$ for the donor-acceptor distance, respectively. These constraints for $\mathrm{H}$-bonds did not lead to an increase in residual constraints violation. Backbone torsion angle constraints for $\alpha$ and $\zeta$ were restricted to a range of $-115^{\circ} /-15^{\circ}$ and $-95^{\circ}-35^{\circ}$, respectively, in accordance to the observed ${ }^{31} \mathrm{P}$ chemical shifts (41-43). Glycosidic torsion angles for all guanines were kept in a range of $-190^{\circ} /-140^{\circ}$ (anti conformation), whereas a range of $-157^{\circ} / 117^{\circ}$ (anti conformation) was used for thymine residues. The remaining backbone torsion angles $(\beta, \gamma$ and $\varepsilon$ ) were kept in range of $-210^{\circ} /-150^{\circ}, 30^{\circ} / 80^{\circ}$ and $-180^{\circ} /-140^{\circ}$, consistently with the torsion angles observed in regular A-DNA. The 20 structures with the lowest CYANA target functions were subjected to energy minimization (with no angle constraints) using the conjugate gradient method and the CVFF force field as implemented in the program DISCOVER (Accelrys). During energy minimization, interproton distances and H-bond constraints involving G-tetrads were used with a force constant of 20 and $100 \mathrm{kcal} \mathrm{mol}^{-1} \AA^{-2}$, respectively. Illustrations of structures were generated with the INSIGHTII program (Accelrys). All the calculations have been performed on a SGI Octane workstation.

The final set of coordinates has been deposited in the Protein Data Bank (accession code 1S9L).

\section{Circular dichroism and CD melting experiments}

CD samples of Q-LNA and natural counterparts [d(TGGGT) $]_{4}$ and $[\mathrm{r}(\mathrm{UGGGU})]_{4}$ were prepared at a concentration of $2.5 \times$ $10^{-5} \mathrm{M}$, by using the buffer solution used for NMR experiments: $10 \mathrm{mM} \mathrm{KH}{ }_{2} \mathrm{PO}_{4}, 70 \mathrm{mM} \mathrm{KCl}, 0.2 \mathrm{mM}$ EDTA, pH 7.0. $\mathrm{CD}$ spectra of both quadruplexes and $\mathrm{CD}$ melting curves were registered on a Jasco 715 circular dichroism spectrophot- ometer in a $0.1 \mathrm{~cm}$ pathlength cuvette. For the CD spectra, the wavelength was varied from 220 to $340 \mathrm{~nm}$ at $5 \mathrm{~nm} \mathrm{~min}^{-1}$, and the spectra recorded with a response of $16 \mathrm{~s}$, at $2.0 \mathrm{~nm}$ bandwidth and normalized by subtraction of the background scan with buffer. The temperature was kept constant at $20^{\circ} \mathrm{C}$ with a thermoelectrically controlled cell holder (Jasco PTC-348).

CD melting curves were registered as a function of temperature from 20 to $90^{\circ} \mathrm{C}$ at 261 and $288 \mathrm{~nm}$ (data not shown) for Q-LNA, and $264 \mathrm{~nm}$ for both [d(TGGGT) $]_{4}$ and $[\mathrm{r}(\mathrm{UGGGU})]_{4}$. The $\mathrm{CD}$ data were recordered in the same buffer as used for NMR experiments in a $0.1 \mathrm{~cm}$ pathlength cuvette with a scan rate of $10^{\circ} \mathrm{C} \mathrm{h}^{-1}$.

\section{RESULTS}

\section{Proton assignment of Q-LNA resonances}

The NMR sample (see Materials and Methods) was annealed for 5-10 $\mathrm{min}$ at $80^{\circ} \mathrm{C}$ and slowly cooled down to room temperature. The ${ }^{1} \mathrm{H}-\mathrm{NMR}$ spectrum (Fig. 2) of Q-LNA shows eight sharp resonances. Three resonances in the low field region correspond to the three imino protons involved in Hoogsteen hydrogen bonds. In addition, five base protons and two methyl resonances (data not shown) are observed in the respective high field regions. The appearance of the ${ }^{1} \mathrm{H}-\mathrm{NMR}$ spectrum already suggests the absence of conformational heterogeneity and a high symmetry of the Q-LNA.

Assignment of Q-LNA resonances started with the analysis of the TOCSY spectrum. All $\mathrm{H}^{\prime}{ }^{\prime}$ and $\mathrm{H}_{2}^{\prime}$ resonances in a given sugar were identified from this spectrum. Some of the $\mathrm{H} 2^{\prime} / \mathrm{H}^{\prime}{ }^{\prime}$ crosspeaks were not observed in the TOCSY spectrum (Fig. 3) due to the small $J$-coupling constant. Hence, information from the NOESY spectra was used to complete the assignment of the $\mathrm{H}^{\prime}$, and to fully assign the $\mathrm{H} 5^{\prime} / \mathrm{H}^{\prime \prime}, \mathrm{H} 6^{\prime} / \mathrm{H}^{\prime \prime}$, aromatic and exchangeable protons. The NOE pattern of Q-LNA turned out to be similar to that observed for other parallel quadruplex structures (Fig. 4) (26,44-47) and, hence, the assignment was accomplished mainly following the standard procedures (48). However, it is interesting to note that $\mathrm{H}^{\prime} / \mathrm{H}^{\prime \prime}$ protons in the $\mathrm{C}^{\prime} / \mathrm{C}^{\prime}$ ' linker of the LNA sugar form an independent spin system; therefore, the assignment of the pertinent signals was accomplished by the analysis of the NOESY spectrum, on the base of their strong intranucleotide crosspeaks to $\mathrm{H}^{\prime}$ (Fig. 5) (49). The $\mathrm{H} 5^{\prime} / \mathrm{H} 5^{\prime \prime}$, instead, are scalar coupled with the adjacent ${ }^{31} \mathrm{P}$ of the phosphate backbone. Therefore, the assignment of these protons has been achieved by the analysis of both NOESY spectrum (containing crosspeaks between $\mathrm{H} 5^{\prime} / \mathrm{H} 5^{\prime \prime}$ and $\mathrm{H} 8 / \mathrm{H} 6$ resonances of the same residue) and heteronuclear ${ }^{1} \mathrm{H}-{ }^{31} \mathrm{P}$ COSY (see below).

Observation of an unbroken path of NOE connectivities between H8/H6 protons and the ribose protons of the adjacent nucleoside at the $5^{\prime}$ side, suggests that the backbone conformation resembles that of a duplex DNA or RNA and that the Q-LNA adopts a right-handed helix structure (26-27,44-47). Furthermore, the lack of strong NOEs between $\mathrm{G} \mathrm{H} 8$ and the $\mathrm{H}^{\prime}{ }^{\prime}$ of the same residue, in comparison with those observed between $\mathrm{G} \mathrm{H8}$ and its ribose $\mathrm{H} 2$ ', suggests that all $\mathrm{G}$ residues are in an anti glycosidic conformation (26-27,45-47). 


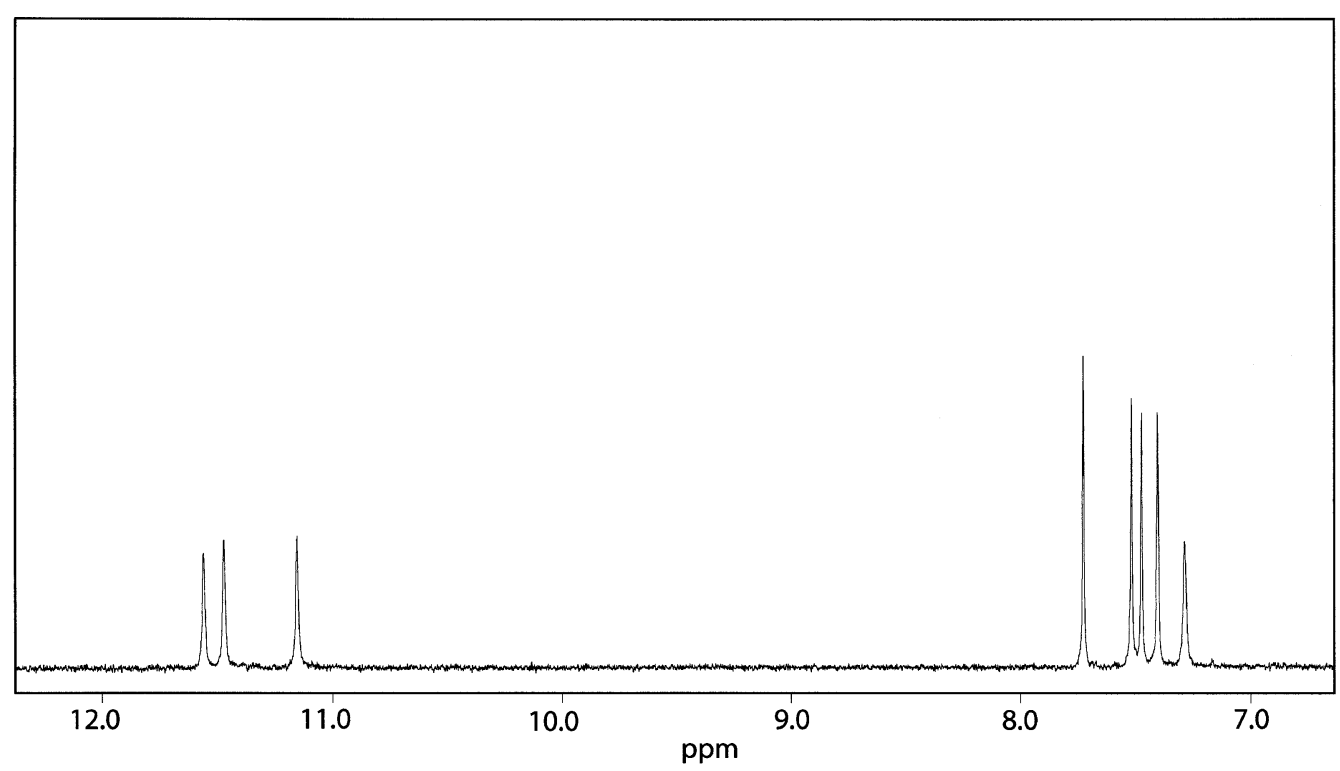

Figure 2. Imino and base proton NMR spectrum (6.5-12.5 p.p.m., $500 \mathrm{MHz})$ of Q-LNA $1.80 \mathrm{mM}$ in $0.6 \mathrm{ml}\left(\mathrm{H}_{2} \mathrm{O} / \mathrm{D}_{2} \mathrm{O} 9: 1\right)$ buffer solution having $10 \mathrm{mM}$ $\mathrm{KH}_{2} \mathrm{PO}_{4}, 70 \mathrm{mM} \mathrm{KCl}, 0.2 \mathrm{mM}$ EDTA, pH 7.0 at $27^{\circ} \mathrm{C}$. The guanine imino protons involved in G-tetrad formation resonate between 11.0 and 12.0 p.p.m. The narrow non-exchangeable base protons resonate between 7.0 and 8.0 p.p.m.

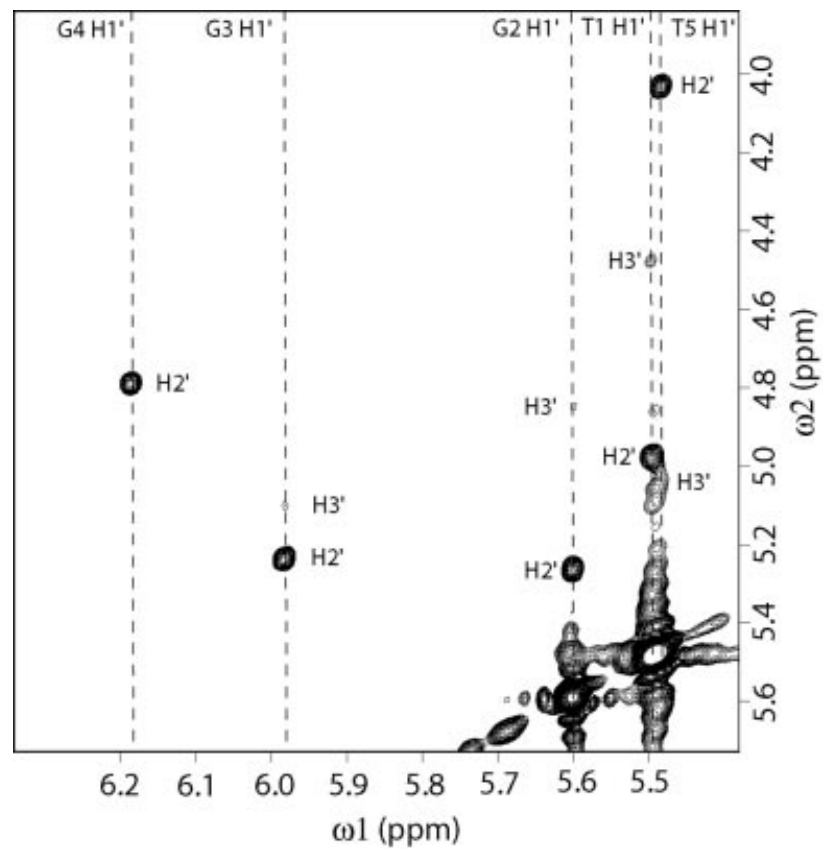

Figure 3. An expanded TOCSY (500 MHz, $120 \mathrm{~ms}$ mixing time) contour plot correlating sugar $\mathrm{H}^{\prime}$ resonances (depicted by vertical dashed lines) and the corresponding $\mathrm{H}^{\prime} / \mathrm{H}^{\prime}$ resonances (between 4.0 and 5.5 p.p.m. and between 4.4 and 5.1 p.p.m., respectively) of Q-LNA $1.80 \mathrm{mM}$ in $0.6 \mathrm{ml}$

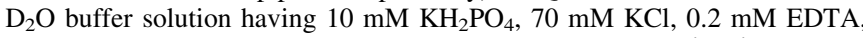
$\mathrm{pH} 7.0$ at $27^{\circ} \mathrm{C}$. Since the $J$-coupling constants between $\mathrm{H}^{\prime} / \mathrm{H}^{\prime}$ are small, some crosspeaks are weaker or missing.

No NOE crosspeaks correlating exchangeable protons could be observed since imino-imino contacts within each tetrad involve magnetically equivalent protons due to the 4-fold symmetry of Q-LNA. Nevertheless, the assignment of the three imino protons has been accomplished through the

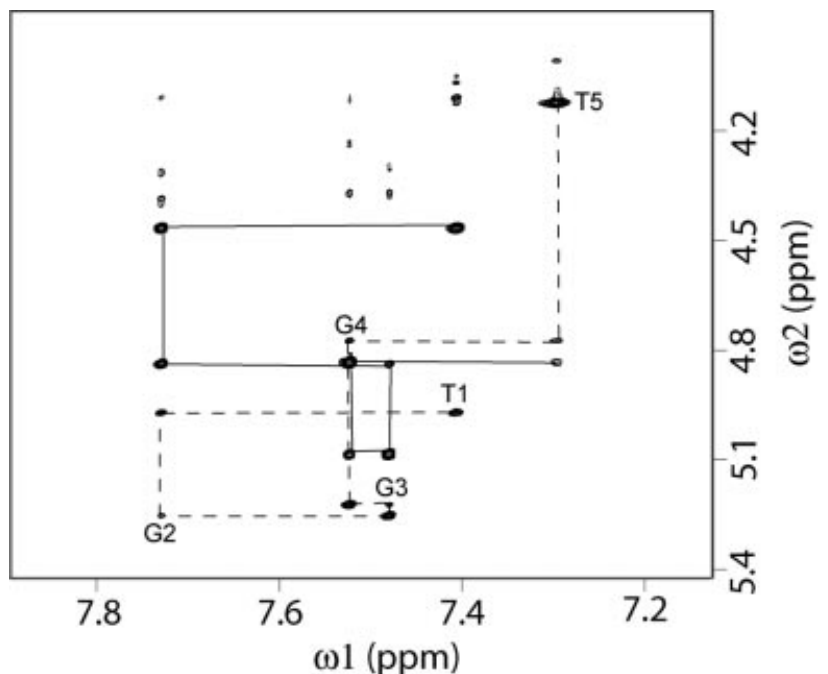

Figure 4. Expanded region of a NOESY spectrum $(750 \mathrm{MHz}, 180 \mathrm{~ms}$ mixing time) containing aromatic and $\mathrm{H}^{\prime} / \mathrm{H}^{\prime}$ (dashed and continous lines, respectively) sequential crosspeaks of Q-LNA $1.80 \mathrm{mM}$ in $0.6 \mathrm{ml} \mathrm{D}_{2} \mathrm{O}$

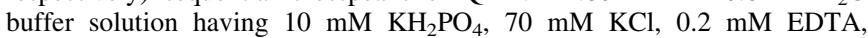
$\mathrm{pH} 7.0$ at $27^{\circ} \mathrm{C}$.

connectivities between imino protons of $\mathrm{G} 2$ and $\mathrm{G} 4$ residues with the adjacent methyls of T1 and T5 residues, respectively.

\section{Phosphorus assignments}

The proton decoupled phosphorus spectrum of Q-LNA in $\mathrm{D}_{2} \mathrm{O}$ at $27^{\circ} \mathrm{C}$ shows that all four ${ }^{31} \mathrm{P}$ signals fall in the region between -1 and -3 p.p.m., characteristic of A-type DNA form (41-43). The 2D proton-detected heteronuclear ${ }^{1} \mathrm{H}_{-}{ }^{31} \mathrm{P}$ COSY allowed the assignment of each phosphorus resonance by its correlation to the respective $5^{\prime}$-coupled $\mathrm{H} 3^{\prime}$ proton and 
Table 1. Proton chemical shifts (750 MHz) of Q-LNA in $10 \mathrm{mM} \mathrm{KH}_{2} \mathrm{PO}_{4}, 70 \mathrm{mM} \mathrm{KCl}, 0.2 \mathrm{mM}$ EDTA $(\mathrm{pH} 7.0, \mathrm{~T}=300 \mathrm{~K})$

\begin{tabular}{lllllllll}
\hline Base $\left(5^{\prime}-3^{\prime}\right)$ & $\mathrm{H} 8 / \mathrm{H} 6$ & $\mathrm{H} 1^{\prime}$ & $\mathrm{H} 2^{\prime}$ & $\mathrm{H} 3^{\prime}$ & $\mathrm{H} 6^{\prime} / \mathrm{H} 6^{\prime \prime}$ & $\mathrm{H} 5^{\prime} / \mathrm{H} 5^{\prime \prime}$ & $\mathrm{H} 2 / \mathrm{Me}$ & $\mathrm{NH}$ \\
\hline $\mathrm{T}$ & 7.41 & 5.47 & 4.98 & 4.46 & $3.95 / 4.10$ & 4.06 & 1.39 & -1.59 \\
$\mathrm{G}$ & 7.74 & 5.58 & 5.25 & 4.84 & $4.03 / 4.20$ & $4.31 / 4.38$ & -2.85 \\
$\mathrm{G}$ & 7.48 & 5.96 & 5.22 & 5.08 & $4.10 / 4.19$ & $4.30 / 4.37$ & 4.16 & 11.48 \\
$\mathrm{G}$ & 7.52 & 6.16 & 4.77 & 4.83 & 4.11 & $4.23 / 4.37$ & -2.66 \\
$\mathrm{~T}$ & 7.31 & 5.45 & 4.12 & 5.06 & $3.83 / 4.00$ & $4.08 / 4.18$ & 1.65 & -1.53 \\
\hline
\end{tabular}

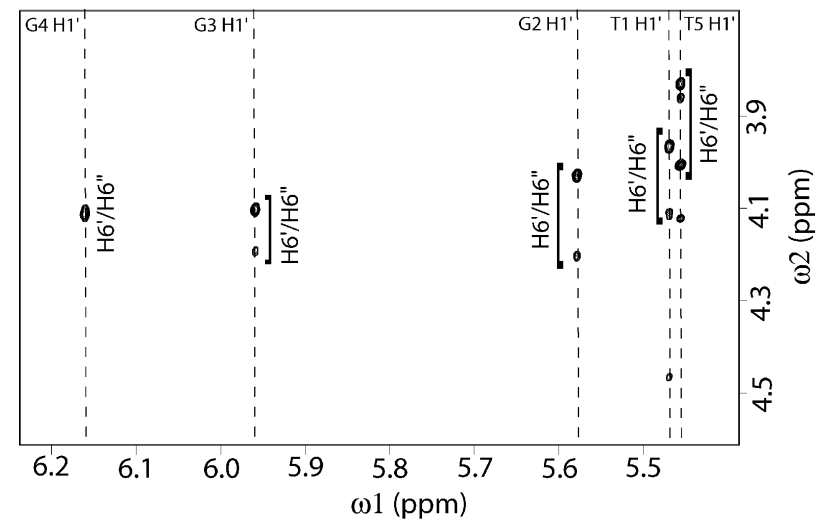

Figure 5. Expanded NOESY (750 MHz, $180 \mathrm{~ms}$ mixing time) contour plots correlating sugar $\mathrm{H}^{\prime}$ ' protons (depicted by vertical dashed lines) and $\mathrm{H}^{\prime} / \mathrm{H} 6$ " resonances (3.8-4.3 p.p.m.) of the $\mathrm{C}^{\prime} / \mathrm{C}^{\prime}$ linker of the LNA sugar of Q-LNA.

3'-coupled H5' and H5" protons. The phosphorus resonances at GT (and TG) steps were slightly different from those observed for the phosphorus at GG steps (see Table 1) indicating structural differences in these two regions.

\section{Experimental restraints and structure calculations}

The crosspeaks in the NOESY spectrum of Q-LNA are well dispersed, thus allowing an easy quantification of the experimental NMR data. From a total of 544 upper distance restraints for Q-LNA, 292 (73 per strand) turned out to be nontrivial and were used in the final calculations. Proton/proton scalar couplings for the definition of the sugar pucker of QLNA have not been measured since X-ray crystallography, NMR spectroscopy and molecular modeling studies have already shown that the LNA sugar is locked in an N-type conformation with a pseudorotation angle of $\mathrm{P}=17^{\circ}(2-4,50$ 53). Constraints for the backbone torsion angles $\alpha$ and $\zeta$ were deduced from the ${ }^{31} \mathrm{P}$ chemical shift. ${ }^{31} \mathrm{P}$ resonates downfield when both $\alpha$ and $\zeta$ are not in the usual gauche ${ }^{-}$conformation. As shown in Table $1,{ }^{31} \mathrm{P}$ chemical shifts of Q-LNA range between -3 and -1 p.p.m., typical for an A-type DNA conformation. Thus, the torsion angles $\alpha$ and $\zeta$ were restricted in range of $-115^{\circ} /-15^{\circ}$ and $-95^{\circ} /-35^{\circ}$, respectively. The backbone torsion angles $\beta$ and $\varepsilon$ were estimated from the scalar coupling measured in the $2 \mathrm{D}$ proton-detected heteronuclear ${ }^{1} \mathrm{H}^{31} \mathrm{P}$ COSY by using the semi-empirical Karplus

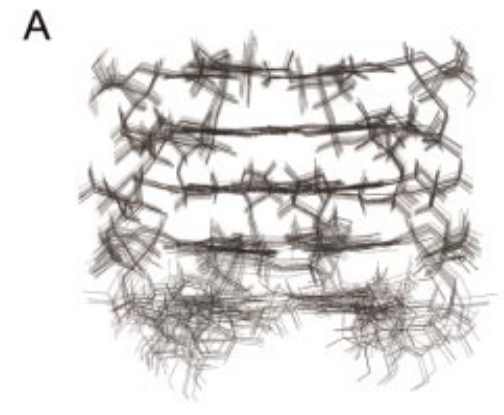

B
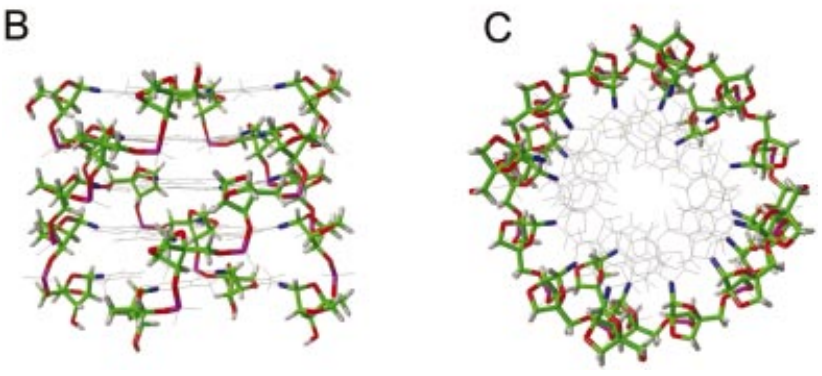

Figure 6. (A) Side view representation of the superimposed 20 best structures of Q-LNA. Side $(\mathbf{B})$ and top $(\mathbf{C})$ view representations of the best NMR structure of Q-LNA. Backbones are depicted in colored 'sticks' (carbons, green; nitrogens, blue; oxygens, red; hydrogens, white), whereas bases in black 'lines'.

equation $(41,54)$. The $\beta$ torsion angles were estimated from ${ }^{31} \mathrm{P}-\mathrm{H} 5^{\prime} / \mathrm{H} 5^{\prime \prime}$ scalar coupling constants, ${ }^{3} J_{\mathrm{P}, \mathrm{H} 5^{\prime}}$ and ${ }^{3} J_{\mathrm{P}, \mathrm{H} 5^{\prime \prime}}$, whereas the $\varepsilon$ torsion angles were estimated from ${ }^{31} \mathrm{P}-\mathrm{H} 3^{\prime}$ scalar coupling constants, ${ }^{3} J_{\mathrm{P}, \mathrm{H} 3^{\prime}}$. As no stereospecific assignments for the $\mathrm{H}^{\prime}$ and $\mathrm{H}^{\prime \prime}$ resonances could be deduced and since the self-cancellation of antiphase splittings of the signal in the $2 \mathrm{D}{ }^{1} \mathrm{H}_{-}{ }^{31} \mathrm{P}$ COSY lead to unreliable measurements for ${ }^{3} J_{\mathrm{P}, \mathrm{H} 5^{\prime}}$ and ${ }^{3} J_{\mathrm{P}, \mathrm{H} 5^{\prime \prime}}$, the $\beta$ and $\varepsilon$ torsion angles were only restricted to very broad ranges consistent with the torsion angles observed in A-type DNA. Due to the lack of $\mathrm{H}^{\prime}$ protons ${ }^{3} J_{\mathrm{H}^{\prime}, \mathrm{H} 5^{\prime}}$ and ${ }^{3} J_{\mathrm{H} 4^{\prime}, \mathrm{H} 5^{\prime \prime}}$ coupling constants were not accessible, and the $\gamma$ torsion angles were restricted in the range observed for A-type DNA. The final structures obtained from calculations were checked for consistency with measured ${ }^{3} J_{\mathrm{P}, \mathrm{H} 3^{\prime}}$ coupling constant.

According to the presence of three imino protons involved in Hoogsteen hydrogen bonds and their symmetry indicated in the ${ }^{1} \mathrm{H}-\mathrm{NMR}$ spectrum, a total of 48 supplementary distance 
Table 2. Experimental constraints and structure statistics of the best 20 structures with the lowest CYANA target functions
Experimental constraints

Total NOEs

NOE-derived constraints used for

structure calculations

Hydrogen bonds

Dihedral angle constraints

Structure statistics

Dyana target function $\left(\AA^{2}\right)$

CVFF energy $(\mathrm{kcal} / \mathrm{mol})$ of the

minimized structures

Total

Van der Waals

Electrostatic

r.m.s. deviations from the mean

structure (§)

All backbone heavy atoms
All heavy atoms
544 (136 per strand) 292 (73 per strand) 96 (24 per strand)

$0.62 \pm 7.43 \times 10^{-3}$

$936 \pm 16$

$111 \pm 4$

$824 \pm 19$ $0.80 \pm 0.30 \AA$
24 (three G-tetrads)

$0.94 \pm 0.31 \AA$

restraints (HN1-O6, N1-O6, HN2-N7, N2-N7) for the 24 hydrogen bonds in the three G-tetrads were included.

From the 200 structures calculated, starting from randomized conformers, the 20 structures with the lowest CYANA (40) target functions (resulting from van der Waals and restraints violations) were selected for final analysis.

The final structures were subjected to a restrained energy minimization (no angle constraints were used) using the CVFF forcefield. Average RMSD values of $0.94 \pm 0.31$ and $0.80 \pm$ $0.30 \AA$ for the backbone and all heavy atoms, respectively, were obtained from their superimposition (Fig. 6A). These data suggest that the structure adopted by Q-LNA is consistent with the experimentally determined restraints. A summary of the experimental constraints and structural statistics for the best 20 structures is reported in Table 2 . The molecule resulted to be parallel stranded, with a 4-fold rotational symmetry, showing a right-handed helicity and possessing three welldefined G-tetrads. The appearances of the structures (Fig. 6B and C) clearly indicate that the G-tetrads are planar and parallel to each other. This observation was also confirmed by CURVES $(55,56)$ analysis performed on the structure possessing the lowest energy after minimization. Buckle, Tilt and Roll angles were calculated to be very close to $0^{\circ}$, whereas Propeller angles were calculated to be very close to $180^{\circ}$ (see Tables 3 and 4). Twist angles for the core GGG segment resulted to be $\sim 30^{\circ}$. The Axial rise (the distance between two adjacent tetrads) for Q-LNA is on average $3.41 \AA$ (see Table 4). Backbone conformation resembles the A-type DNA form (Table 5) and the groove width for Q-LNA (measured as the shortest P-P distance) varies between 8.13 and $8.30 \AA$ in the central core (GGG) of the quadruplex. The LNA quadruplex structure is better defined at the $5^{\prime}$ edge than at $3^{\prime}$ end. This is mainly due to the fact that the $5^{\prime}$ edge of Q-LNA is characterized by a higher number of intra- and inter-strand NOEs.

\section{CD spectroscopy of Q-LNA}

CD spectra for Q-LNA and the natural counterparts $[\mathrm{d}(\mathrm{TGGGT})]_{4}$ and $[\mathrm{r}(\mathrm{UGGGU})]_{4}$ have been acquired at $20^{\circ} \mathrm{C}$ and are reported in Figure 7. The spectrum of Q-LNA is characterized by two positive bands at 261 and $288 \mathrm{~nm}$, and by
Table 3. Shear, stretch, stagger, buckle, propeller, opening of the bestminimized structure

\begin{tabular}{lrrrrrr}
\hline & Shear & Stretch & Stagger & Buckle & Propeller & Opening \\
\hline Strand 1/2 & & & & & & \\
T1 & -10.13 & -1.37 & 0.01 & -6.27 & 165.29 & -88.37 \\
G2 & -6.91 & 1.84 & 0.04 & -9.93 & 165.81 & -89.69 \\
G3 & -6.88 & 1.83 & 0.05 & -5.63 & 163.37 & -90.15 \\
G4 & -6.87 & 1.87 & -0.04 & -8.87 & 172.28 & -90.46 \\
T5 & -5.09 & 0.35 & 0.14 & 10.69 & 186.83 & -74.27 \\
Average value & -7.18 & 1.10 & 0.04 & -4.00 & 170.71 & -86.59 \\
Strand 1/3 & & & & & & \\
T1 & -10.23 & -1.39 & -0.02 & -5.62 & 165.61 & 91.56 \\
G2 & -7.01 & 1.85 & 0.03 & -8.90 & 165.73 & 90.33 \\
G3 & -6.98 & 1.83 & 0.05 & -4.35 & 163.12 & 89.89 \\
G4 & -7.00 & 1.88 & 0.00 & -7.68 & 171.97 & 89.65 \\
T5 & -5.20 & 1.34 & 0.21 & 11.92 & 186.57 & 105.61 \\
Average value & -7.29 & 1.10 & 0.05 & -2.93 & 170.60 & 93.41 \\
Strand 1/4 & & & & & & \\
T1 & -10.02 & -1.21 & 0.06 & -6.86 & 163.53 & -0.33 \\
G2 & -6.93 & 1.90 & 0.06 & -10.62 & 166.59 & 0.03 \\
G3 & -6.93 & 1.86 & 0.07 & -5.49 & 165.63 & 0.02 \\
G4 & -6.90 & 1.88 & 0.10 & -5.84 & 177.61 & 0.08 \\
T5 & -2.79 & 0.11 & 0.06 & 7.30 & 188.91 & 0.31 \\
Average value & -6.71 & 0.91 & 0.07 & -4.30 & 172.45 & 0.02 \\
\hline
\end{tabular}

Table 4. Rise, tilt, roll, twist of the best-minimized structure

\begin{tabular}{lrrrr}
\hline & Rise & \multicolumn{1}{l}{ Tilt } & Roll & Twist \\
\hline Strand 1 & & & & \\
T1/G2 & 3.66 & -0.69 & 1.43 & 44.05 \\
G2/G3 & 3.40 & 2.66 & -0.61 & 28.83 \\
G3/G4 & 3.50 & -0.05 & 5.85 & 27.73 \\
G4/T5 & 3.18 & 6.67 & 5.58 & 32.01 \\
Average value & 3.44 & 2.15 & 3.06 & 33.16 \\
Strand 2 & & & & \\
T1/G2 & 3.64 & 1.97 & -0.90 & 45.37 \\
G2/G3 & 3.39 & -1.65 & -1.96 & 29.30 \\
G3/G4 & 3.59 & 3.20 & 2.84 & 28.04 \\
G4/T5 & 2.99 & -12.8 & 8.90 & 15.82 \\
Average value & 3.40 & -2.32 & -2.22 & 29.63 \\
Strand 3 & & & & \\
T1/G2 & 3.61 & 1.59 & -1.29 & 45.28 \\
G2/G3 & 3.37 & -1.89 & -2.13 & 29.28 \\
G3/G4 & 3.55 & 3.28 & 2.78 & 27.97 \\
G4/T5 & 2.97 & -12.84 & 8.94 & 16.05 \\
Average value & 3.38 & -2.47 & 2.08 & 29.65 \\
Strand 4 & & & & \\
T1/G2 & 3.67 & 2.07 & 1.64 & 43.69 \\
G2/G3 & 3.39 & -2.46 & -0.48 & 28.84 \\
G3/G4 & 3.47 & -0.30 & 5.92 & 27.67 \\
G4/T5 & 3.22 & -6.38 & 5.63 & 31.79 \\
Average value & 3.44 & -1.77 & 3.18 & 33.00 \\
\hline
\end{tabular}

a negative band at $239 \mathrm{~nm}$. The spectrum of Q-LNA slightly differs from those of $[\mathrm{d}(\mathrm{TGGGT})]_{4}$ and $[\mathrm{r}(\mathrm{UGGGU})]_{4}$, in that, besides the typical bands of a parallel stranded quadruplex (44), a shoulder at $288 \mathrm{~nm}$ is present. Also this unusual band, however, can be confidentially associated with the presence of the quadruplex structure since the CD melting profile recorded at $288 \mathrm{~nm}$ perfectly matches that at $261 \mathrm{~nm}$.

In order to estimate the thermal stability, the Q-LNA was subjected to both melting and annealing CD experiments. Additionally, a comparison with the natural counterparts 
Table 5. Chi, gamma, delta, epsilon, zeta, alpha, beta angles of the best-minimized structure

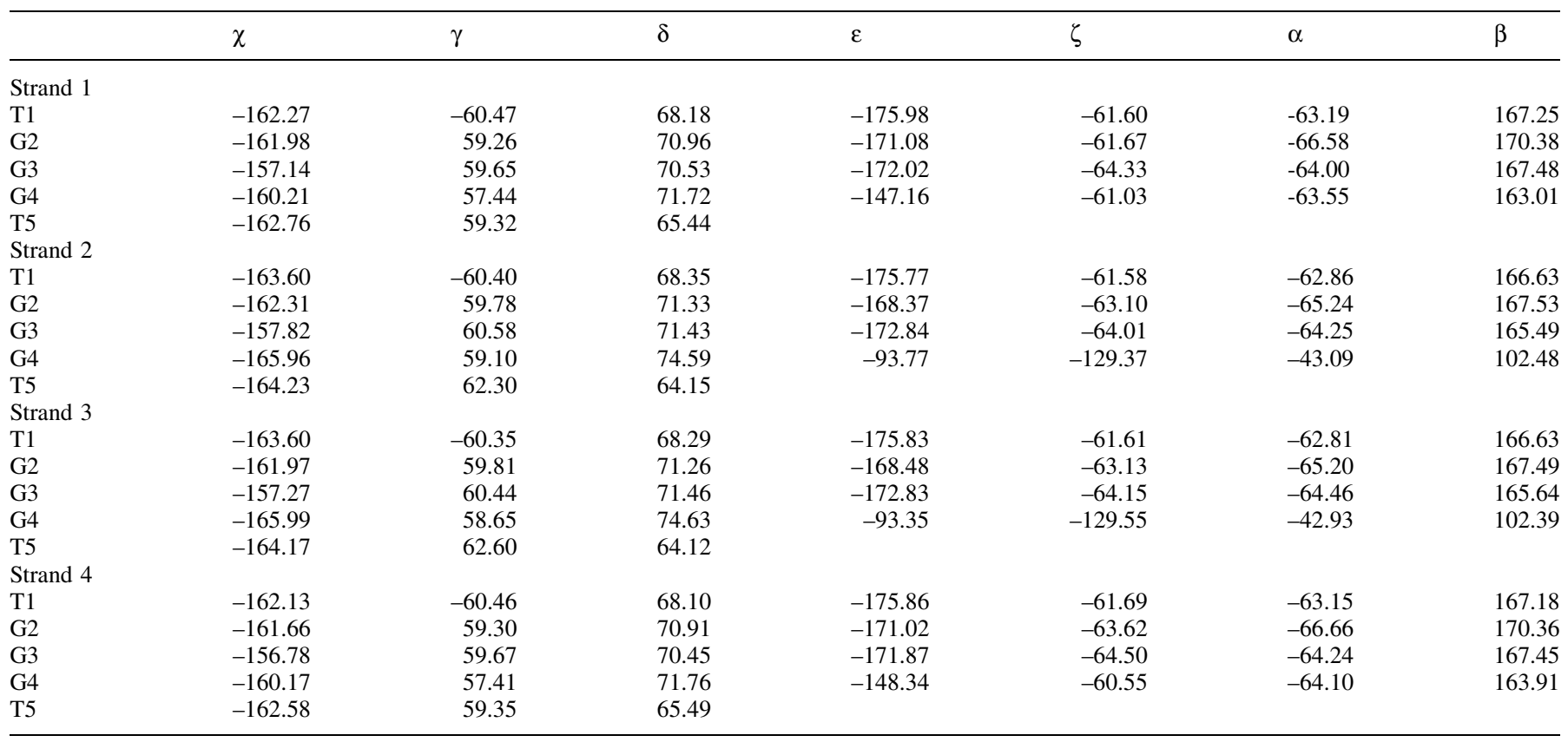

$[\mathrm{d}(\mathrm{TGGGT})]_{4}$ and $[\mathrm{r}(\mathrm{UGGGU})]_{4}$, under the same experimental conditions, was performed. From the results shown in Figure 7, a significant hysteresis emerges for each oligonucleotide when comparing annealing and melting curves. Furthermore, the former do not show a well defined sigmoid shape and, in the case of $[\mathrm{d}(\mathrm{TGGGT})]_{4}$ and $[\mathrm{r}(\mathrm{UGGGU})]_{4}$, the apparent annealing $T_{\mathrm{m}}$ could not be appraised. From the melting curves, midpoint $T_{\mathrm{m}}$ values of 66,46 and $67^{\circ} \mathrm{C}$ could be measured for Q-LNA, [d(TGGGT) $]_{4}$ and $[\mathrm{r}(\mathrm{UGGGU})]_{4}$, respectively. Thus, whereas there is no significant difference with the $T_{\mathrm{m}}$ observed for $[\mathrm{r}(\mathrm{UGGGU})]_{4}$, a considerable increase $\left(20^{\circ} \mathrm{C}\right)$ in the melting temperature and, hence, in the thermal stability could be determined for the Q-LNA when compared with the DNA counterpart.

\section{DISCUSSION}

The NMR data indicate that, under the experimental condition described here, Q-LNA forms a single well-defined quadruplex structure consisting of three G-tetrads and possessing a 4-fold symmetry with all strands parallel to each other in analogy with the unmodified quadruplex $[\mathrm{d}(\mathrm{TGGGT})]_{4}$. The 2D NOESY spectrum clearly indicates that Q-LNA adopts a right-handed helix. All bases are in anti conformation and all Gs form Hoogsteen hydrogen bonds involved in G-tetrads. A direct comparison of the most representative structures of QLNA (the one with lowest energy after minimization), and the already reported NMR structures of the RNA parallel quadruplex $[(\mathrm{UGGGGU})]_{4}$ (PDB code: $\left.1 \mathrm{RAU}\right)$ and of the DNA parallel quadruplex [d(TTGGGGT) $]_{4}$ (PDB code: 139D) has been accomplished by analyzing the helix parameters by CURVES $(55,56)$. These data clearly suggest that the structure of Q-LNA differs from the others. The base stacking within all quadruplexes is quite similar and is mostly intra-strand; in fact, in any of the three structures, the five-membered rings of the guanines overlap with the six-membered rings of the 5 '-flanking bases, whereas the imino and amino protons overlap with the six-membered rings of the 3'-flanking bases. This observation was confirmed by the fact that all three structures have basically the same base Twist angles for the core GGG segment $\left(\sim 30^{\circ}\right)$. Thus, the three structures seem to have very similar geometry for the bases, while the major differences could be observed at the backbone region. They are characterized by different Axial rise. The rise value for Q-LNA (3.41 $\AA$ ) was calculated to be in between the values calculated for $[(\mathrm{UGGGGU})]_{4}(3.94 \AA)$ and $[\mathrm{d}(\mathrm{TTGGGGT})]_{4}$ $\left(3.30 \AA\right.$ A). Since all LNA nucleotides are locked in a $\mathrm{C}^{\prime}$-endo (N-type) conformation, the backbone structure of the each strand in the Q-LNA could resemble A-type rather than B-type DNA as observed, for example, in other parallel DNA quadruplex structures (44-47). In fact, the backbone torsion angles for both Q-LNA (Table 5) and [(UGGGGU) $]_{4}$ fall in the same range of A-type DNA, while they differ in comparison with $[\mathrm{d}(\mathrm{TTGGGGT})]_{4}$ especially for $\delta$ and $\zeta$ torsion angles. Particularly, $\delta$ and $\zeta$ fall in the range of $60 \%$ $100^{\circ}$ and $-100^{\circ} /-50^{\circ}$, respectively, for the former structures, while they range between $130^{\circ} / 160^{\circ}$ and $-100^{\circ} /-140^{\circ}$ for [d(TTGGGGT) $]_{4}$. The groove width for Q-LNA, calculated to be between 8.13 and $8.30 \AA$ in the central core (GGG), is the smallest of the three compared structures.

The thermal stabilities of Q-LNA, [d(TGGGT) $]_{4}$ and $[\mathrm{r}(\mathrm{UGGGU})]_{4}$ were compared by $\mathrm{CD}$ melting and annealing experiments. The observed hysteresis indicates that, in spite of the very low scan rate used in the experiments, the cooling and heating curves are not in a thermodynamic equilibrium, i.e. the temperature change is faster than the rate at which the folding/ unfolding processes reach a new equilibrium. On the other hand, the hysteresis reflects a difference in kinetics of 

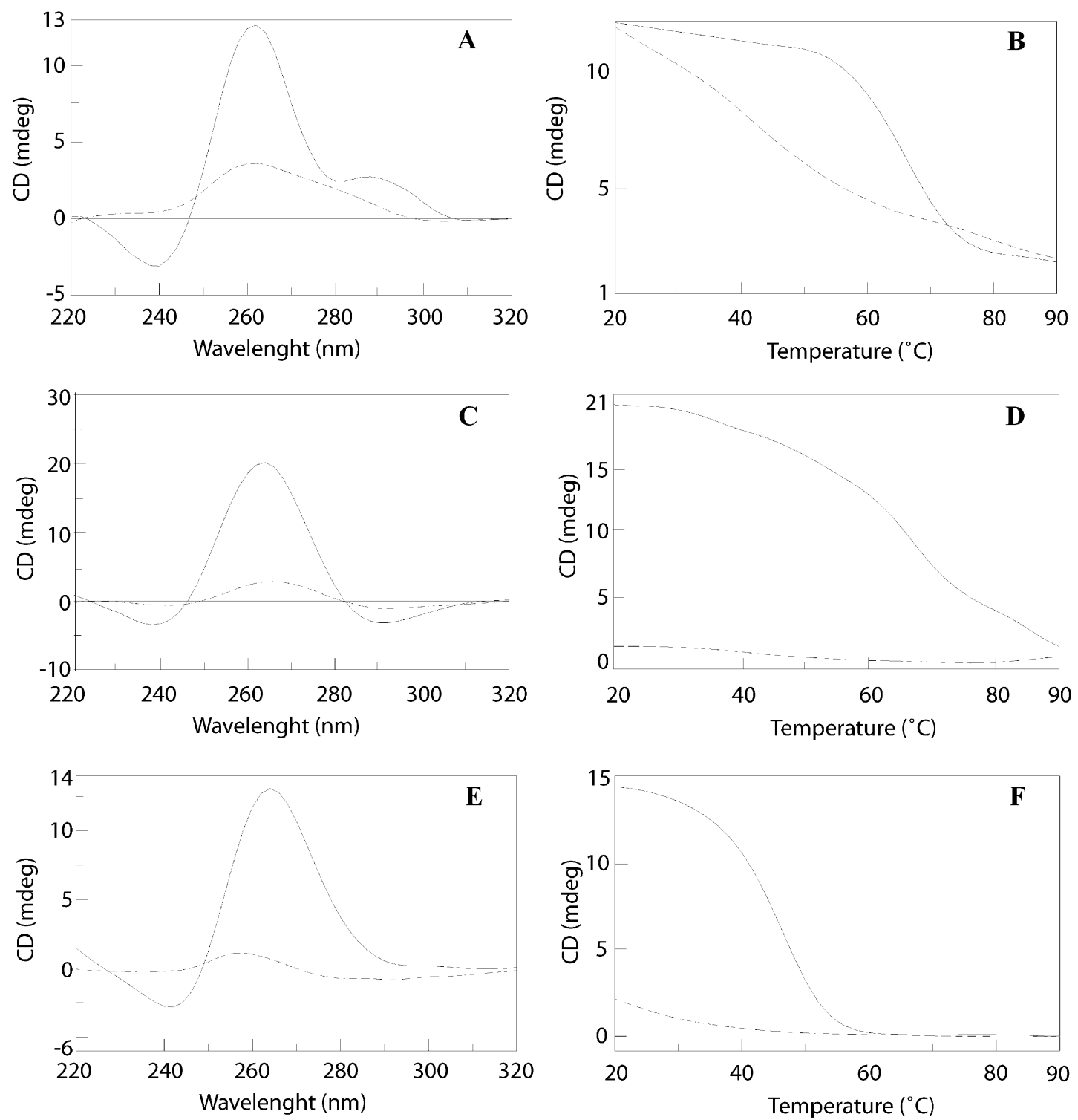

Figure 7. CD spectra of Q-LNA (A), $[\mathrm{r}(\mathrm{UGGGU})]_{4}(\mathbf{C})$ and $[\mathrm{d}(\mathrm{TGGGT})]_{4}(\mathbf{E})$ at $20^{\circ} \mathrm{C}$ (continous lines) and $90^{\circ} \mathrm{C}$ (dashed lines). Melting (continous lines) and annealing (dashed lines) CD experiments of Q-LNA (B), [r(UGGGU) $]_{4}(\mathbf{D})$ and $[\mathrm{d}(\mathrm{TGGGT})]_{4}(\mathbf{F})$.

association versus dissociation, as is often found for multistrand nucleic acid structures. Even if a more detailed physicochemical study has not been yet accomplished, it is not unreasonable to hypothesize a considerably slower kinetics of association versus dissociation, considering that, in principle, the former process requires the organization and the assemblage of four separate strands. For this reason we estimate the melting profiles a better descriptive picture of the situation at the equilibrium than the annealing curves.

It is noteworthy that, according to CD melting experiments, both Q-LNA and $[\mathrm{r}(\mathrm{UGGGU})]_{4}$ are characterized by higher thermal stabilities than $[\mathrm{d}(\mathrm{TGGGT})]_{4}$. In analogy to that reported for LNA containing duplex structures (5-7), it has to be assumed that the huge increase in the melting temperature for Q-LNA is, at least partly, entropy related, since the LNA sugars are pre-organized for the formation of the quadruplex (as also indicated by the smaller hysteresis; Fig. 7B). As a matter of fact, no increment of base stacking or hydrogen bonding is observed in the structure of Q-LNA in comparison with other parallel DNA quadruplexes (44-47). However, the N-type nature of LNAs sugar conformation itself might play a role in stabilizing Q-LNA. This is suggested by the similar thermal stability of the RNA quadruplex, which, on the other hand, could be ascribed to the $\mathrm{H}$-bonds formed by $\mathrm{OH}$ groups at the $\mathrm{C}^{\prime}$ ' positions (27), as well. In order to further clarify this topic, a more in depth study will be performed in our laboratory.

The structures reported are, to the best of our knowledge, the first example of LNA quadruplex structures. These results, particularly when viewed in the context of other recent findings about LNA, serve to underscore further the polymorphic nature of LNAs. Furthermore, the many advantageous characteristics of LNA usage, such as a very good water solubility, easy handling, synthesis using conventional 
phosphoramidite chemistry, high thermal stability and the markedly increased stability towards nuclease digestion, demonstrate that LNAs might amplify the range of applicability of synthetic oligonucleotides as aptamers or catalysts. Numerous examples exist where nucleic acids (including quadruplex structures) have been selected as aptamers for proteins or small molecules (33). Thus, the new structural features found here could be used for the creation of novel aptameric and catalytic nucleic acids in order to provide novel structural motifs for ligand-binding pockets with diverse molecular recognition capabilities that would not be present using native RNA/DNA sequences.

\section{SUPPLEMENTARY MATERIAL}

Supplementary Material is available at NAR Online.

\section{ACKNOWLEDGEMENTS}

This work is supported by Italian M.U.R.S.T. (P.R.I.N. 2002 and 2003) and Regione Campania (L.41, L.5). The authors are grateful to 'Centro Ricerche Interdipartimentale di Analisi Strumentale', C.R.I.A.S., for supplying NMR facilities. Luca Cozzuto is acknowledged for his kind collaboration.

\section{REFERENCES}

1. Koshkin,A.A., Rajwanshi,V.K. and Wengel,J. (1998) Novel convenient syntheses of LNA [2.2.1]bicyclo nucleosides. Tetrahedron Lett., 39, 4381-4384.

2. Obika,S., Nanbu,D., Hari,Y., Andoh,J., Morio,K., Doi,T. and Imanishi,T. (1998) Stability and structural features of the duplexes containing nucleoside analogs with a fixed N-type conformation, $2^{\prime}-\mathrm{O}, 4^{\prime}-\mathrm{C}$ methyleneribonucleosides. Tetrahedron Lett., 39, 5401-5404.

3. Wengel,J. (1998) Synthesis of 3'-C- and 4'-C-branched oligonucleotides and the development of locked nucleic acid (LNA). Acc. Chem. Res., 32, 301-310.

4. Singh,S.K., Nielsen,P., Koshkin,A.A. and Wengel,J. (1998) LNA (locked nucleic acids): synthesis and high-affinity nucleic acid recognition. Chem. Commun., 4, 455-456.

5. Nielsen,K.E., Singh,S.K., Wengel,J. and Jacobsen,J.P. (2000) Solution structure of an LNA hybridized to DNA: NMR study of the d(CTLGCTLTLCTLGC):d(GCAGAAGCAG) duplex containing four locked nucleotides. Bioconjugate Chem., 11, 228-238.

6. Bondensgaard,K., Petersen,M., Singh,S.K., Rajwanshi,V.K., Kumar,R., Wengel,J. and Jacobsen,J.P. (2000) Structural studies of LNA:RNA duplexes by NMR: conformations and implications for RNase $\mathrm{H}$ activity. Chem. Eur. J., 6, 2687-2695.

7. Braasch,D.A. and Corey,D.R. (2001) Locked nucleic acid (LNA): finetuning the recognition of DNA and RNA. Chem. Biol., 8, 1-7.

8. Koller,E., Gaarde,W.A. and Monia,B.P. (2000) Elucidating cell signaling mechanisms using antisense technology. Trends Pharm. Sci., 21, 142-148.

9. Braasch,D.A. and Corey,D.R. (2002) Novel antisense and peptide nucleic acid strategies for controlling gene expression. Biochemistry, 41, 4503-4510.

10. Opalinska,J.B. and Gewirtz,A.M. (2002) Nucleic-acid therapeutics: basic principles and recent applications. Nature Rev. Drug Discov., 1, 503-514.

11. Arzumanov,A., Walsh,A.P., Rajwanshi,V.K., Kumar,R., Wengel,J. and Gait,M.J. (2001) Inhibition of HIV-1 Tat-dependent trans activation by steric block chimeric 2'-O-methyl/LNA oligoribonucleotides. Biochemistry, 40, 14645-14654.

12. Vester,B., Lundberg,L.B., Sorensen,M.D., Babu,B.R., Douthwaite,S. and Wengel,J. (2002) LNAzymes: incorporation of LNA-type monomers into DNAzymes markedly increases RNA cleavage. J. Am. Chem. Soc., 124, 13682-13683.
13. Fluiter,K., ten Asbroek,A.L.M.A., de Wissel,M.B., Jakobs,M.E., Wissenbach,M., Olsson,H., Olsen,O., Oerum,H. and Baas,F. (2003) In vivo tumor growth inhibition and biodistribution studies of locked nucleic acid (LNA) antisense oligonucleotides. Nucleic Acids Res., 31, 953-962.

14. Obika,S., Hari,Y., Ken-Ichiro,M. and Imanishi,T. (2000) Triplex formation by an oligonucleotide containing conformationally locked C-nucleoside, 5-(2-O,4-C-methylene- $\beta$-D-ribofuranosyl)oxazole. Tetrahedron Lett., 41, 221-224.

15. Torigoe,H., Hari,Y., Sekiguchi,M., Obika,S. and Imanishi,T. (2001) 2'-O,4'-C-Methylene bridged nucleic acid modification promotes pyrimidine motif triplex DNA formation at physiological $\mathrm{pH}$ : thermodynamic and kinetic studies. J. Biol. Chem., 276, 2354-2360.

16. Elayadi,A.N., Braasch,D.A. and Corey,D.R. (2002) Implications of highaffinity hybridization by locked nucleic acid oligomers for inhibition of human telomerase. Biochemistry, 41, 9973-9981.

17. Bielinska,A., Shivdasani,R.A., Zhang,L.Q. and Nabel,G.J. (1990) Regulation of gene expression with double-stranded phosphorothioate oligonucleotides. Science, 250, 997-1000.

18. Crinelli,R., Bianchi,M., Gentilini,L. and Magnani,M. (2002) Design and characterization of decoy oligonucleotides containing locked nucleic acids. Nucleic Acids Res., 30, 2435-2443.

19. Belotserkovskii,B., Reddy,G. and Zarling,D. (2000) Locked nucleic acid hybrids and their uses in modulating genetic processes. Patent Application WO 2000-US10909 20000421.

20. Henderson,E., Hardin,C.C., Walk,S.K., Tinoco,I. and Blackburn E.H.,Jr (1987) Telomeric DNA oligonucleotides form novel intramolecular structures containing guanine-guanine base pairs. Cell, 51, 899-908.

21. Macaya,R.F., Schultze,P., Smith,F.W., Roe,J.A. and Feigon,J. (1993) Thrombin-binding DNA aptamer forms a unimolecular quadruplex structure in solution. Proc. Natl Acad. Sci. USA, 334, 3745-3749.

22. Wang,Y. and Patel,D.J. (1995) Solution structure of the Oxytricha telomeric repeat $\mathrm{d}\left[\mathrm{G}_{4}\left(\mathrm{~T}_{4} \mathrm{G}_{4}\right)_{3}\right]$ G-tetraplex. J. Mol. Biol., 251, 76-94.

23. Schultze,P., Flint,W.S. and Feigon,J. (1994) Refined solution structure of the dimeric quadruplex formed from the Oxytricha telomeric oligonucleotide d(GGGGTTTTGGGG). Structure, 2, 221-233.

24. Smith,F.W. and Feigon,J. (1992) Quadruplex structure of Oxytricha telomeric DNA oligonucleotides. Nature, 356, 164-168.

25. Aboul-ela,F., Murchie,A.I.H. Norman,D.G. and Lilley,D.M.J. (1994) Solution structure of a parallel-stranded tetraplex formed by $\mathrm{d}\left(\mathrm{TG}_{4} \mathrm{~T}\right)$ in the presence of sodium ions by nuclear magnetic resonance spectroscopy. J. Mol. Biol., 243, 458-471.

26. Patel,P.K., Koti,A.S. R. and Hosur,R.V. (1999) NMR studies on truncated sequences of human telomeric DNA: observation of a novel A-tetrad. Nucleic Acids Res., 27, 3836-3843.

27. Cheong,C. and Moore,P.B. (1992) Solution structure of an unusually stable RNA tetraplex containing G- and U-quartet structures. Biochemistry, 31, 8406-8414.

28. Blackburn,E.H. and Szostak,J.W. (1984) The molecular structure of centromeres and telomeres. Annu. Rev. Biochem., 53, 163-194.

29. Blackburn,E.H. (1994) Telomeres: no end in sight. Cell, 77, 621-623.

30. Sen,D. and Gilbert,W. (1988) Formation of parallel four-stranded complexes by guanine-rich motifs in DNA and its implications for meiosis. Nature, 334, 364-366.

31. Simonsson,T., Pecinka,P. and Kubista,M. (1998) DNA tetraplex formation in the control region of c-myc. Nucleic Acids Res., 26, 1167-1172.

32. Wells,R.D., Collier,D.A., Hanvey,J.C., Shimizu,M. and Wohlrab,D. (1988) The chemistry and biology of unusual DNA structures adopted by oligopurine.oligopyrimidine sequences. FASEB J., 2, 2939-2949.

33. Osborne,S.E., Matsumura,I. and Ellington,A.D. (1997) Aptamers as therapeutic and diagnostic reagents: problems and prospects. Curr. Opin. Chem. Biol., 1, 5-9.

34. Esposito,V., Randazzo,A., Messere,A., Galeone,A., Petraccone,L. Giancola,C., Piccialli,G. and Mayol,L. (2003) Synthesis and structural characterization of PNA-DNA quadruplex forming chimeras. Eur. J. Org. Chem., 17, 3364-3371.

35. Datta,B., Schmitt,C. and Armitage,B.A. (2003) Formation of a $\mathrm{PNA}_{2}-\mathrm{DNA}_{2}$ hybrid quadruplex. J. Am. Chem. Soc., 125, 4111-4118.

36. Sklenar,V., Piotto,M., Leppik,R. and Saudek,V. (1993) Gradient-tailored water suppression for $1 \mathrm{H}-15 \mathrm{~N}$ HSQC experiments optimized to retain full sensitivity. J. Magn. Reson., 102, 241-245. 
37. Jeener,J., Meier,B.H., Bachmann,P. and Ernst,R.R. (1979) Investigation of exchange process by two-dimensional NMR spectroscopy. J. Chem. Phys., 71, 4546-4553.

38. Braunschweiler,L. and Ernst,R.R. (1983) Coherence transfer by isotropic mixing: application to proton correlation spectroscopy. J. Magn. Reson. 53, 521-528.

39. Marion,D. and Wuthrich,K. (1983) Application of phase sensitive twodimensional correlated spectroscopy (COSY) for measurements of proton-proton spin-spin coupling constants in proteins. Biochem. Biophys. Res. Commun., 113, 967-974.

40. Guntert,P., Mumenthaler,C. and Wuthrich,K. (1997) Torsion angle dynamics for NMR structure calculation with the new program DYANA J. Mol. Biol., 273, 283-298.

41. Wijmenga,S.S. and van Buuren,B.N.M. (1998), The use of NMR methods for conformational studies of nucleic acids. Prog. Nuclear Magnetic Resonance Spectroscopy, 32, 287-387.

42. Gorenstein,D.G. (1984) In Gorenstein,D.G. (ed.), Phosphorus-31 NMR: Principles and Applications. Academic Press, New York.

43. Roongta,V.A., Jones,C.R. and Gorenstein,D.G. (1990) Effect of distortions in the deoxyribose phosphate backbone conformation of duplex oligodeoxyribonucleotide dodecamers containing GT, GG, GA, $\mathrm{AC}$ and GU base-pair mismatches on 31P NMR spectra. Biochemistry, 29, 5245-5258.

44. Jin,R., Gaffney,B.L., Wang,C., Jones,R.A. and Breslauer,K.J. (1992) Thermodynamics and structure of a DNA tetraplex: a spectroscopic and calorimetric study of the tetramolecular complexes of $\mathrm{d}\left(\mathrm{TG}_{3} \mathrm{~T}\right)$ and $\mathrm{d}\left(\mathrm{TG}_{3} \mathrm{~T}_{2} \mathrm{G}_{3} \mathrm{~T}\right)$. Proc. Natl Acad. Sci. USA, 89, 8832-8836.

45. Wang,Y. and Patel,D.J. (1993) Solution structure of a parallel-stranded G-quadruplex DNA. J. Mol. Biol., 234, 1171-1183.

46. Aboul-ela,F., Murchie,A.I.H. and Lilley,D.M.J. (1992) NMR study of a parallel-stranded tetraplex formation by the hexadeoxynucleotide $\mathrm{d}\left(\mathrm{TG}_{4} \mathrm{~T}\right)$. Nature, 360, 280-282.
47. Patel,P.K. and Hosur,R.V. (1999) NMR observation of T-tetrads in a parallel stranded DNA quadruplex formed by Saccharomyces cerevisiae telomere repeats. Nucleic Acids Res., 27, 2457-2464.

48. Wüthrich,K. (1986) NMR of Proteins and Nucleic Acids. Wiley, New York.

49. Bondensgaard,K., Petersen,M., Singh,S.K., Rajwanshi,V.K., Kumar,R., Wengel,J. and Jacobsen,J.P. (2000) Structural study of LNA:RNA duplexes by NMR: conformations and implications for RNase $\mathrm{H}$ activity. Chem. Eur. J., 15, 2687-2695.

50. Koshkin,A.A., Singh,S.K., Nielsen,P., Rajwanshi,V.K., Kumar,R., Meldgaard,M., Olsen,C.E. and Wengel,J. (1998) LNA (locked nucleic acids): synthesis of the adenine, cytosine, guanine, 5-methylcytosine, thymine and uracil bicyclonucleoside monomers, oligomerization and unprecedented nucleic acid recognition. Tetrahedron, 54, 3607-3630.

51. Singh,S.K. and Wengel,J. (1998) Universality of LNA-mediated highaffinity nucleic acid recognition. Chem. Comm., 12, 1247-1248.

52. Koshkin,A.A., Nielsen,P., Meldgaard,M., Rajwanshi,V.K., Singh,S.K. and Wengel,J. (1998) LNA (Locked Nucleic Acid): an RNA mimic forming exceedingly stable LNA:LNA duplexes. J. Am. Chem. Soc., 120, $13252-13253$.

53. Kumar,R., Singh,S.K., Koshkin,A.A., Rajwanshi,V.K., Meldgaard,M. and Wengel,J. (1998) The first analogs of LNA (locked nucleic acids): phosphorothioate-LNA and 2'-thio-LNA. Bioorganic Med. Chem. Lett., 8, 2219-2222.

54. Altona,C. (1982) Conformational analysis of nucleic acids. Determination of backbone geometry of single-helical RNA and DNA in aqueous solution. J. Royal Netherlands Chem. Soc., 101, 413-433.

55. Lavery,R. and Sklenar,H. (1988) The definition of generalized helicoidal parameters and of axis curvature for irregular nucleic acids. J. Biomol. Struct. Dyn., 6, 63-91.

56. Lavery,R. and Sklenar,H. (1989) Defining the structure of irregular nucleic acids: conventions and principles. J. Biomol. Struct. Dyn., 6, 655-667. 